\title{
Quantitative Proteomics of Enriched Esophageal and Gut Tissues from the Human Blood Fluke Schistosoma mansoni Pinpoints Secreted Proteins for Vaccine Development
}

Leandro X. Neves ${ }^{1}$, R. Alan Wilson ${ }^{2}$, Philip Brownridge ${ }^{3}$, Victoria M. Harman ${ }^{3}$, Stephen W. Holman ${ }^{3}$, Robert J. Beynon ${ }^{3}$, Claire E. Eyers ${ }^{3}$, Ricardo DeMarco ${ }^{4}$ and William Castro-Borges ${ }^{1 *}$

${ }^{1}$ Departamento de Ciências Biológicas, Universidade Federal de Ouro Preto, Campus Morro do Cruzeiro, Ouro Preto, Minas Gerais, Brasil; ${ }^{2}$ Centre for Immunology and Infection, Department of Biology, University of York, Heslington, York, United Kingdom; ${ }^{3}$ Centre for Proteome Research, Department of Biochemistry, Institute of Integrative Biology, University of Liverpool, Liverpool, L69 7ZB, United Kingdom; ${ }^{4}$ Instituto de Física de São Carlos, Universidade de São Paulo, São Carlos, Brasil.

*Correspondence should be addressed to:

William de Castro Borges, Instituto de Ciências Exatas e Biológicas II, sala 58, Laboratório de Enzimologia e Proteômica. Universidade Federal de Ouro Preto, Campus Morro do Cruzeiro. Ouro Preto - Minas Gerais Brasil. CEP: 35.400-000. Phone: +55 313559 1705; FAX: +55 313559 1680;

e-mail: wborges@ufop.edu.br

\section{Summary}

Supplementary Material S1_Expanded methodology of shotgun proteomic analyses

Page S-2

Supplementary Material S2_EsoCAT construct, heterologous expression and

Page S-5

monitored transitions

Supplementary Material S3_Identification and relative quantification of MEG proteins Page S-9

Supplementary Material S4_Descriptive statistics of QconCAT assay and

Page S-12

protein copy per cell estimation

Supplementary Material S5_Abundance of gastrodermis markers

Page S-14

and statistical differences

\section{Spreadsheets associated to the manuscript}

Supplementary Table S1_Label-free quantification and tissue signatures.xlsx

Supplementary Table S2_QconCAT absolute quantification.xlsx 


\section{Supplementary Material S1_Expanded methodology of shotgun proteomic analyses}

\section{UltiMate 3000 UHPLC coupled to Q Exactive HF mass spectrometer}

Digested samples (700 ng) were injected in the system at a $5 \mu \mathrm{L} \cdot \mathrm{min}^{-1}$ flow rate of aqueous loading phase $\left(2 \%\right.$ acetonitrile $-\mathrm{MeCN}, 0.05 \%$ trifluoroacetic acid) and trapped onto a PepMap100 $\mathrm{C}_{18}$ column (5 cm x $300 \mu \mathrm{m}, 5 \mu \mathrm{m}, 100 \AA \AA$; Dionex) for washing over $7 \mathrm{~min}$. The peptide mixture was then resolved on the analytical column Easy-Spray PepMap $C_{18}$ RSLC (75 $\mu \mathrm{m}$ x $50 \mathrm{~cm}, 2 \mu \mathrm{m}, 100$ $\AA$; Dionex), conditioned at $40{ }^{\circ} \mathrm{C}$, using a gradient from $96.2 \%$ solvent $\mathrm{A}(0.1 \%$ formic acid in water) / 3.8\% solvent B ( $80 \%$ water, $20 \% \mathrm{MeCN}, 0.1 \%$ formic acid) to $60 \% \mathrm{~A} / 40 \% \mathrm{~B}$ over $95 \mathrm{~min}$, at a flow rate of $300 \mathrm{nl} \cdot \mathrm{min}^{-1}$. System was regenerated with $99 \% \mathrm{~B}$ and re-equilibrated for $15 \mathrm{~min}$ before next injection. Q Exactive HF was operated in positive mode using a Top16 method. Survey scans were acquired at resolution of 60,000 FWHW (at $200 \mathrm{~m} / \mathrm{z}$ ), mass range of 350-2,000 $\mathrm{m} / \mathrm{z}$, automatic gain control (AGC) of 3E6 and maximum injection time (IT) of $100 \mathrm{~ms}$. Precursor ions with charge state $+2,+3,+4$ and +5 were isolated within a $1.2 \mathrm{~m} / \mathrm{z}$ window and activated by higherenergy collisional dissociation (HCD) with stepped normalized collision energy (SNCE) of 28-30V. Automatic gain control was set to 1E5 with maximum injection time of $45 \mathrm{~ms}$. Product ions spectra were acquired at 30,000 FWHW. Dynamic exclusion of 40 seconds was active. Biological replicates (i) and (ii) of ESO and BE samples were analysed in triplicates.

\section{UltiMate 3000 UHPLC coupled to Orbitrap Fusion mass spectrometer}

The biological replicate (ii) was also analysed in the Orbitrap Fusion platform with minor changes in the LC method described above, namely: flow rate for desalting of peptides at $9 \mu \mathrm{L} \cdot \mathrm{min}^{-1}$ and elution gradient from $96.2 \% \mathrm{~A} / 3.8 \% \mathrm{~B}$ to $50 \% \mathrm{~A} / 50 \% \mathrm{~B}$ over $90 \mathrm{~min}$, at constant temperature of $30^{\circ} \mathrm{C}$. A TopSpeed method with 3 seconds cycles was used. Precursor spectra were acquired at 120,000 FWHW (at $200 \mathrm{~m} / \mathrm{z}$ ), mass range of 300-1,750 m/z, AGC target 2E5 and IT $50 \mathrm{~ms}$. Precursor ions with charge states $+2,+3,+4$ and +5 were selected for dissociation (AGC target $1 \mathrm{E} 4$ and IT of 35 
ms). Samples were analysed using two methods differing on the activation methods: a) collisional induced dissociation, CID (35\%), and b) higher-energy collisional dissociation, HCD (SNCE 30\% $\pm 5 \%$ ). Product ions were detected using the rapid scan rate of the ion trap. Dynamic exclusion of $60 \mathrm{~s}$ was activated.

\section{UltiMate 3000 UHPLC coupled to Q Exactive mass spectrometer (geLC-MS/MS)}

HE (Oesophagus with oral sucker attached) and BE fragments were quickly washed in $2 \mathrm{~mL}$ of icecold PBS, pH 7.4, for RNAlater removal. Samples were homogenised in PBS by sonication on icebath for 5 cycles of 30 seconds pulses at 30\% amplitude, $10 \%$ duty cycle and output control of 5 (450-Sonifier Analog Cell Disruptor; Branson Ultrasonics), with 1 min intervals between series. Total protein concentration was determined using PIERCE ${ }^{\mathrm{TM}}$ BCA Protein Assay Kit (Thermo Scientific) by interpolation from a BSA standard curve, according to manufacturer's instructions.

Twenty micrograms of each sample were loaded onto a 10\% SDS-PAGE (1:29, 30\% acrylamide/bisacrylamide) and separated until the upper band $(225 \mathrm{kDa})$ of the pre-stained Full-Range Rainbow ${ }^{\mathrm{TM}}$ MW marker (GE Healthcare, USA) had entered the separating gel ( 15min). With the gel stained, a vertical strip with the lane's length was excised, chopped into $1 \mathrm{~mm}^{2}$ pieces, and subjected to in-gel tryptic digestion. Briefly, gel fragments were washed in ultrapure water followed by reduction (50 $\mathrm{mM}$ DTT at $65^{\circ} \mathrm{C}$ for $\left.30 \mathrm{~min}\right)$ and alkylation $(100 \mathrm{mM}$ iodoacetamide, at room temperature for $1 \mathrm{~h})$. Gel pieces were washed in $20 \mathrm{mM} \mathrm{AMBIC} / 50 \% \mathrm{MeCN}$ for $3 \times 20 \mathrm{~min}$ each and dried using a Savant ${ }^{\mathrm{TM}}$ SpeedVac concentrator (Savant, Thermo Scientific). Gel slices were rehydrated in $15 \mu \mathrm{L}$ of 40mM AMBIC containing MS grade trypsin (Promega) at $0.04 \mu \mathrm{g} / \mu \mathrm{L}$ and incubated at $37{ }^{\circ} \mathrm{C}$ for 16h. After recovering the digestion supernatant, the gel pieces were extracted in $50 \% \mathrm{MeCN}$ solution containing $0.1 \%$ TFA. Peptides were then concentrated to dryness using SpeedVac and resuspended in $2 \% \mathrm{MeCN}$ containing $0.1 \%$ TFA.

Approximately $4 \mu \mathrm{g}$ of digested proteins were injected in the system at a $5 \mu \mathrm{L} \cdot \mathrm{min}^{-1}$ flow rate of loading phase (2\% MeCN, $0.05 \%$ trifuoloacetic acid) and trapped for 3 min onto a PepMap100 $\mathrm{C}_{18}$ 
column (75 $\mu \mathrm{m}$ i.d. $\times 2 \mathrm{~cm}, 3 \mu \mathrm{m}, 100 \AA$; Dionex $)$ for washing. The peptide mixture was resolved on the Acclaim PepMap100 C18 RSLC RSLC column $(75 \mu \mathrm{m}$ i.d. $\times 15$ cm, $2 \mu \mathrm{m}, 100 \AA$; Dionex $)$ using a gradient from $96.0 \%$ solvent $\mathrm{A} / 4.0 \%$ solvent B to $70 \% \mathrm{~A} / 30 \% \mathrm{~B}$, over $180 \mathrm{~min}$, following a ramp to $55 \%$ B over 60 min. System was regenerated with $90 \%$ B and re-equilibrated for $15 \mathrm{~min}$ before next injection. Chromatographic separation was carried out at a flow rate of $300 \mathrm{nl} \cdot \mathrm{min}^{-1}$ and $40{ }^{\circ} \mathrm{C}$.

Q Exactive was operated in positive mode using a Top12 method. Survey scans were acquired at resolution of 70,000 FWHW (at $200 \mathrm{~m} / \mathrm{z}$ ), mass range of $300-2,000 \mathrm{~m} / \mathrm{z}$, automatic gain control (AGC) of 3E6 and maximum injection time (IT) of $100 \mathrm{~ms}$. Precursor ions with charge state $\geq+2$ were isolated within a $2 \mathrm{~m} / \mathrm{z}$ window and activated by higher-energy collisional dissociation (HCD) with normalized collision energy (NCE) of 30V. Automatic gain control was set to 2E5 with maximum injection time of $150 \mathrm{~ms}$. Product ions spectra were acquired at 17,500 FWHW (at 200 $m / z)$. Dynamic exclusion of 60 seconds was active. Single runs of ESO and BE were used to build a sample specific spectral library to support the EsoCAT design and provide preliminary evidences of proteins exclusive to the schistosome esophagus. 


\section{Supplementary Material S2_EsoCAT construct, heterologous expression and monitored transitions}

Nineteen proteotypic sequences represented from T4 to T40 tryptic fragments. Blue and red squares indicate lysine and arginine in P1 position, respectively. Blank gaps comprised the three $\mathrm{N}$ - and Cterminal amino acids found flanking the sequence of endogenous worm oesophageal proteins. GluFibrinopeptide B (T2) was used to accurately determine the EsoCAT concentration spiked in the samples. Hexa-histidine (HisTag; T43) was included for affinity purification.

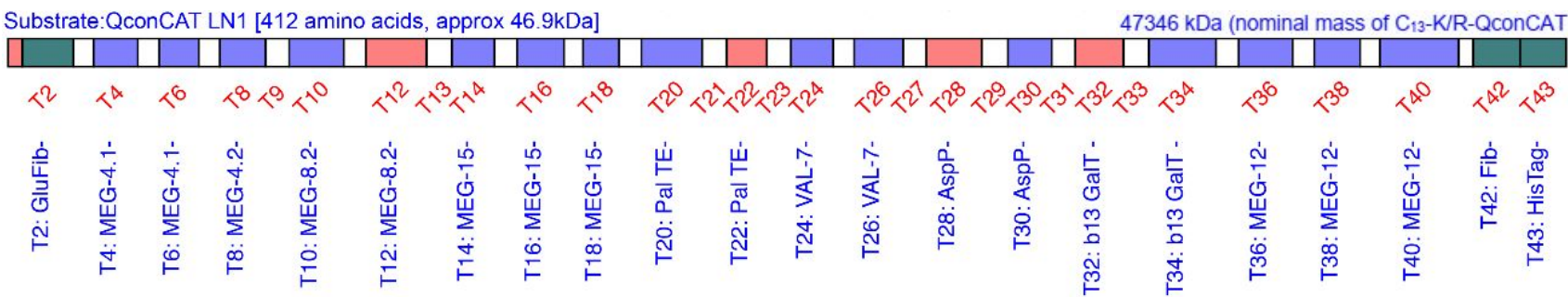

>EsoCAT_Glufib : MEG-4.1 : MEG-4.1 : MEG-4.2 : MEG-8.2 : MEG-8.2 : MEG-15 : MEG-15 :

MEG-15 : Palthio : Palthio : VAL-7 : VAL-7 : Asp-protease : Asp-protease : B-1,3-GalTase : B-

1,3-GalTase : MEG-12 : MEG-12 : MEG-12 : Fib

MAGREGVNDNEEGFFSARGLAKPKSIADIFLINKPKVPLTPKQINDGTSDKPKSAIPHKQLEEEQNPFHKL

SEMNKFNSIFGEEEYNPPKDSDVNKMNTTPVHQEEPSFWRVMWTLKTTLSHHNTVPAKTTVQIKTIEFS

QNENLLKLATVGKLSHHNTVPAKTTVFSKVGTVVSIGGPQQGIFGIPKCINILKLATAIQNEIPRTYVLDKVF

VVCNYGPGGKYANGSKWTYVGQSIAFVPKVVQTIRINSLVIPNQAFGEMRNIFEMRNIFSLDYFTSKYDG

FTKNAENPFDSPYYRVYLCPKQVPLYLFIDDDYDLVPKNVIQPKAYGIWSLFSYFYKTFVGLKENYEQQLQ

QPKAYGGLRKFHIVFFCGENYEQQLQQPKAYGKGVNDNEEGFFSARLAAALEHHHHHH

\section{Heterologous expression of EsoCAT}
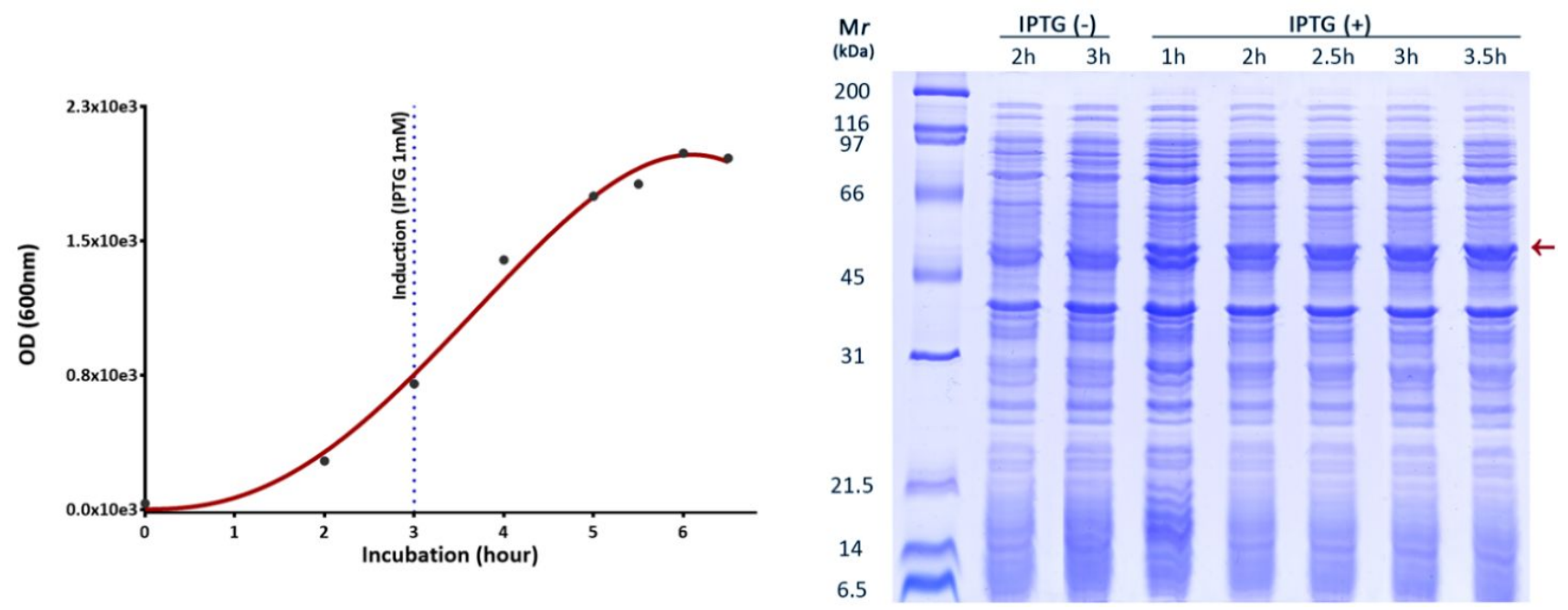

*Visualization of the EsoCAT protein (arrow at $\sim 47 \mathrm{kDa}$ ) expressed after IPTG induction. 
HisTrap $^{\text {TM }}$ affinity purification of EsoCAT

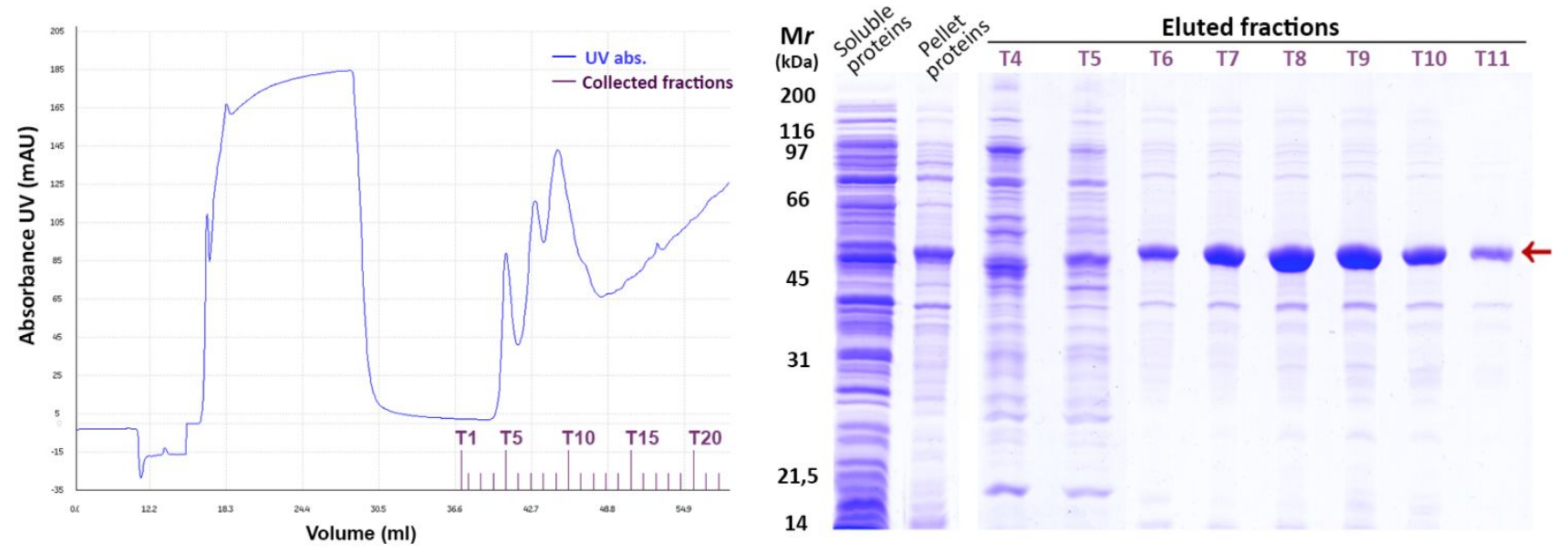

*Visualization of the EsoCAT protein (arrow at $\sim 47 \mathrm{kDa}$ ) enriched in the eluate fractions. 


\section{List of transitions monitored by SRM-MS/MS}

\begin{tabular}{|c|c|c|c|c|c|c|c|}
\hline GeneDB accession & Description & Peptide & $\begin{array}{c}\text { PEAKS score or } \\
\text { CONSeQuence } \\
\text { score (1-4) } \\
\end{array}$ & $\begin{array}{c}\text { QconCAT } \\
\text { tryptic } \\
\text { fragment }\end{array}$ & $\begin{array}{l}\text { Precursor } \\
(m / z)\end{array}$ & $z$ & Product ions $(\mathrm{m} / \mathrm{z})$ \\
\hline \multirow[t]{2}{*}{ Smp_163630 } & \multirow[t]{2}{*}{ MEG-4.1 } & " SIADIFLINKPK & 62.42 & T4 & 453.6063 & +3 & $\begin{array}{c}\mathrm{y} 7 \text { (859.54), y6 (712.47), y5 } \\
(599.38), \text { y4 (486.30) }\end{array}$ \\
\hline & & QINDGTSDKPK & 62.02 & $\mathrm{~T} 6$ & 401.5386 & +3 & $\begin{array}{c}\mathrm{y} 7(732.38), \mathrm{y} 4(487.28), \mathrm{y} 3 \\
(372.26)\end{array}$ \\
\hline Smp_085840 & MEG-4.2 & QLEEEQNPFHK & 43.23 & $\mathrm{~T} 8$ & 699.8360 & +2 & $\begin{array}{c}\mathrm{y} 9 \text { (1157.52), y8 (1028.47), y7 } \\
(899.43)\end{array}$ \\
\hline \multirow[t]{3}{*}{ Smp_172180 } & \multirow[t]{3}{*}{ MEG-8.2 } & FNSIFGEEEYNPPK & 69.04 & $\mathrm{~T} 10$ & 835.8885 & +2 & $\begin{array}{c}\text { y10 (1209.54), y9 (1062.47) } \\
\text { y3 (341.21), b3 (349.15) }\end{array}$ \\
\hline & & MNTTPVHQEEPSFWR & 4 & $\mathrm{~T} 12$ & 620.2895 & +3 & $\begin{array}{c}\text { y9 (1215.55), y8 (1078.49), y7 } \\
(950.43), y 5(692.35)\end{array}$ \\
\hline & & $\begin{array}{l}\mathrm{M}[+16] \mathrm{NTTPVHQEEPSFW} \\
\mathrm{R}\end{array}$ & - & T12' & 625.6212 & +3 & $\begin{array}{c}\text { y9 (1215.55), y8 (1078.49), y6 } \\
\text { (821.39) }\end{array}$ \\
\hline Smp_152630 & MEG-12 & AYGIWSLFSYFYK & 61.54 & T36 & 822.9085 & +2 & $\begin{array}{c}\text { y9 }(1240.60), y 8(1054.52), y 7 \\
(967.49), y 6(854.40), y 5 \\
(707.33)\end{array}$ \\
\hline$S m p \_152630.1$ & $\begin{array}{l}\text { Proteoform 1, } \\
M E G-12\end{array}$ & ENYEQQLQQPK & 1 & $\mathrm{~T} 38$ & 702.8413 & +2 & $\begin{array}{c}\text { y8 (998.52), y7 (869.48), y6 } \\
(741.42), \text { y5 (613.36) }\end{array}$ \\
\hline$S m p \_152630.2$ & $\begin{array}{l}\text { Proteoform 2, } \\
M E G-12\end{array}$ & $\begin{array}{l}\text { FHIVFFCGENYEQQLQQP } \\
\mathrm{K}\end{array}$ & - & $\mathrm{T} 40$ & 804.7196 & +3 & $\begin{array}{c}\text { y8 (998.52), y5 (613.36), b3 } \\
(398.21), \text { b5 (644.35) }\end{array}$ \\
\hline \multirow[t]{3}{*}{ Smp_010550 } & \multirow[t]{3}{*}{ MEG-15 } & TTLSHHNTVPAK & 68.19 & T14 & 435.9018 & +3 & $\begin{array}{c}\text { y7 (766.42), y6 (629.36), y3 } \\
(315.20)\end{array}$ \\
\hline & & TIEFSQNENLLK & 65.90 & T16 & 718.3750 & +2 & $\begin{array}{c}\text { y10 (1221.61), y9 (1092.56), } \\
\text { y8 (945.49) }\end{array}$ \\
\hline & & LSHHNTVPAK & 67.90 & $\mathrm{~T} 18$ & 368.5367 & +3 & $\begin{array}{c}\text { y7 (766.42), } \mathrm{y}^{++}(495.75) \\
\mathrm{y} 7^{++}(383.71)\end{array}$ \\
\hline Smp_242990 & VAL-7 & VFVVC[+57]NYGPGGK & 90.14 & $\mathrm{~T} 24$ & 648.8239 & +2 & y9 (951.43), y8 (852.36), y5 \\
\hline
\end{tabular}




\begin{tabular}{|c|c|c|c|c|c|c|c|}
\hline & & & & & & & $(415.22), \mathrm{y} 4(358.20)$ \\
\hline & & WTYVGQSIAFVPK & 61.74 & $\mathrm{~T} 26$ & 748.4008 & +2 & $\begin{array}{c}\text { y11 (1208.66), y10 (1045.60), } \\
\text { y9 (946.53) }\end{array}$ \\
\hline \multirow[t]{2}{*}{ Smp_142970 } & \multirow[t]{2}{*}{$\begin{array}{l}\text { Palmitoyl } \\
\text { thioesterase }\end{array}$} & VGTVVSIGGPQQGIFGIPK & 35.94 & $\mathrm{~T} 20$ & 618.6876 & +3 & $\begin{array}{c}\mathrm{y} 8(859.50), \mathrm{y} 7(731.44), \mathrm{y} 5 \\
(561.33), \mathrm{b} 4(357.21)\end{array}$ \\
\hline & & LATAIQNEIPR & 58.26 & $\mathrm{~T} 22$ & 613.3486 & +2 & $\begin{array}{c}\text { y9 (1041.56), y7 (869.48), y6 } \\
(756.39), \text { y5 (628.34) }\end{array}$ \\
\hline \multirow[t]{3}{*}{ Smp_132480 } & \multirow[t]{3}{*}{$\begin{array}{l}\text { Aspartyl } \\
\text { protease }\end{array}$} & INSLVIPNQAFGEMR & 71.15 & $\mathrm{~T} 28$ & 844.9431 & +2 & $\begin{array}{c}\text { y11 (1261.63), y10 (1162.56), } \\
\text { y9 (1049.48) }\end{array}$ \\
\hline & & INSLVIPNQAFGEM[+16]R & - & $\mathrm{T} 28^{\prime}$ & 852.9405 & +2 & $\begin{array}{c}\text { y10 (1114.56), y9 (1065.47), } \\
\text { y9 (1001.47) }\end{array}$ \\
\hline & & NIFSLDYFTSK & 59.67 & $\mathrm{~T} 30$ & 667.8350 & +2 & $\begin{array}{c}\mathrm{y} 9(1107.53), \mathrm{y} 8(960.46), \mathrm{y} 3 \\
(335.19)\end{array}$ \\
\hline \multirow[t]{4}{*}{ Smp_151220 } & \multirow[t]{2}{*}{$\beta$-1,3-GalTase } & NAENPFDSPYYR & 59.61 & $\mathrm{~T} 32$ & 736.8257 & +2 & $\begin{array}{c}\text { y9 (1158.52), y8 (1044.47), y6 } \\
(800.35), \text { y5 (685.33) }\end{array}$ \\
\hline & & QVPLYLFIDDDYDLVPK & 4 & $\mathrm{~T} 34$ & 685.0225 & +3 & $\begin{array}{l}\text { y11 (1339.64), y10 (1192.57), } \\
\text { b6 (714.41), b7 (861.48) }\end{array}$ \\
\hline & [Glu1]- & \multirow[t]{2}{*}{ EGVNDNEEGFFSAR } & & $\mathrm{T} 2$ & 785.8421 & +2 & y11 (1285.54), y9 (1056.47), \\
\hline & $\begin{array}{l}\text { Fibrinopeptide } \\
\text { B }\end{array}$ & & & & & & \\
\hline
\end{tabular}




\section{Supplementary Material S3_Identification and relative quantification of MEG proteins}

\begin{tabular}{|c|c|c|c|c|c|c|c|c|c|c|c|c|c|}
\hline \multirow[b]{2}{*}{ Description } & \multirow[b]{2}{*}{ Sequence } & \multicolumn{4}{|c|}{ Fusion } & \multicolumn{4}{|c|}{ Q Exactive HF } & \multicolumn{4}{|c|}{ Q Exactive (spectral library) } \\
\hline & & $\begin{array}{l}\text { ESO } \\
\text { area }\end{array}$ & \#ESO & BE area & $\# \mathbf{B E}$ & $\begin{array}{l}\text { ESO } \\
\text { area }\end{array}$ & \#ESO & BE area & $\# \mathbf{B E}$ & $\begin{array}{l}\text { ESO } \\
\text { area }\end{array}$ & \#ESO & BE area & \#BE \\
\hline MEG-3.4 & CEDDCFGVSAITSK & $1.1 \mathrm{E}+05$ & 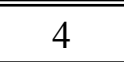 & $0.0 \mathrm{E}+00$ & 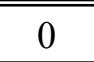 & $0.0 \mathrm{E}+00$ & 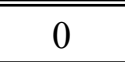 & $0.0 \mathrm{E}+00$ & 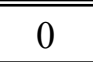 & $0.0 \mathrm{E}+00$ & 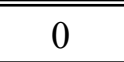 & $0.0 \mathrm{E}+00$ & 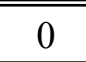 \\
\hline \multirow[t]{14}{*}{ MEG-4.1 (Full) } & FNDVNTINK & $1.1 \mathrm{E}+05$ & 5 & $0.0 \mathrm{E}+00$ & 0 & $0.0 \mathrm{E}+00$ & 0 & $0.0 \mathrm{E}+00$ & 0 & $0.0 \mathrm{E}+00$ & 0 & $0.0 \mathrm{E}+00$ & 0 \\
\hline & FNDVNTINKK & $5.7 \mathrm{E}+05$ & 12 & $0.0 \mathrm{E}+00$ & 0 & $1.1 \mathrm{E}+08$ & 12 & $0.0 \mathrm{E}+00$ & 0 & $6.0 \mathrm{E}+07$ & 2 & $0.0 \mathrm{E}+00$ & 0 \\
\hline & KQFTEEEFSR & $2.4 \mathrm{E}+05$ & 10 & $0.0 \mathrm{E}+00$ & 0 & $0.0 \mathrm{E}+00$ & 0 & $0.0 \mathrm{E}+00$ & 0 & $1.2 \mathrm{E}+07$ & 1 & $0.0 \mathrm{E}+00$ & 0 \\
\hline & QFTEEEFSR & $1.8 \mathrm{E}+06$ & 9 & $0.0 \mathrm{E}+00$ & 0 & $2.0 \mathrm{E}+08$ & 3 & $0.0 \mathrm{E}+00$ & 0 & $1.6 \mathrm{E}+08$ & 2 & $0.0 \mathrm{E}+00$ & 0 \\
\hline & LINSMLK & $\underline{8.1 \mathrm{E}+05}$ & $\underline{10}$ & $0.0 \mathrm{E}+00$ & 0 & $0.0 \mathrm{E}+00$ & 0 & $0.0 \mathrm{E}+00$ & 0 & $0.0 \mathrm{E}+00$ & 0 & $0.0 \mathrm{E}+00$ & 0 \\
\hline & $\overline{\text { EYIEDKNVDIR }}$ & $\overline{0.0 \mathrm{E}+00}$ & $\overline{0}$ & $0.0 \mathrm{E}+00$ & 0 & $2.3 \mathrm{E}+07$ & 5 & $0.0 \mathrm{E}+00$ & 0 & $0.0 \mathrm{E}+00$ & 0 & $0.0 \mathrm{E}+00$ & 0 \\
\hline & TSDTHTIKR & $4.1 \mathrm{E}+04$ & 16 & $0.0 \mathrm{E}+00$ & 0 & $0.0 \mathrm{E}+00$ & 0 & $0.0 \mathrm{E}+00$ & 0 & $0.0 \mathrm{E}+00$ & 0 & $0.0 \mathrm{E}+00$ & 0 \\
\hline & QINDGTSDKTSDTHTIK & $0.0 \mathrm{E}+00$ & 0 & $0.0 \mathrm{E}+00$ & 0 & $\mathrm{NaN}$ & 1 & $0.0 \mathrm{E}+00$ & 0 & $0.0 \mathrm{E}+00$ & 0 & $0.0 \mathrm{E}+00$ & 0 \\
\hline & QINDGTSDKPK & $3.9 \mathrm{E}+05$ & 18 & $0.0 \mathrm{E}+00$ & 0 & $0.0 \mathrm{E}+00$ & 0 & $0.0 \mathrm{E}+00$ & 0 & $8.1 \mathrm{E}+05$ & 1 & $0.0 \mathrm{E}+00$ & 0 \\
\hline & SIADIFLINKPK & $5.5 \mathrm{E}+05$ & 7 & $0.0 \mathrm{E}+00$ & 0 & $9.8 E+07$ & 20 & $0.0 \mathrm{E}+00$ & 0 & $5.5 \mathrm{E}+08$ & 6 & $5.0 \mathrm{E}+06$ & 1 \\
\hline & $\begin{array}{l}\text { SIADIFLINKPKVPLWIVNPLY } \\
\text { YMVEK }\end{array}$ & $0.0 \mathrm{E}+00$ & 0 & $0.0 \mathrm{E}+00$ & 0 & $2.2 \mathrm{E}+07$ & 6 & $0.0 \mathrm{E}+00$ & 0 & $0.0 \mathrm{E}+00$ & 0 & $0.0 \mathrm{E}+00$ & 0 \\
\hline & VPLWIVNPLYYMVEK & $1.0 \mathrm{E}+06$ & 15 & $0.0 \mathrm{E}+00$ & 0 & $4.4 \mathrm{E}+07$ & 16 & $0.0 \mathrm{E}+00$ & 0 & $\underline{4.9 \mathrm{E}+08}$ & $\underline{7}$ & $4.6 \mathrm{E}+06$ & 1 \\
\hline & FVQIMGYLLEDDDTLELNLPK & $0.0 \mathrm{E}+00$ & 0 & $0.0 \mathrm{E}+00$ & 0 & $0.0 \mathrm{E}+00$ & 0 & $0.0 \mathrm{E}+00$ & 0 & $2.0 \mathrm{E}+07$ & $\frac{\overline{2}}{2}$ & $0.0 \mathrm{E}+00$ & 0 \\
\hline & median & $2 E+05$ & & $1 E+00$ & & $1 E+07$ & & $1 E+00$ & & $8 E+05$ & & $1 E+00$ & \\
\hline $\begin{array}{l}\text { MEG-4.1 (Exon } 15 \\
\text { skipped) }\end{array}$ & EYIEDNKK & $3.0 \mathrm{E}+05$ & 3 & $0.0 \mathrm{E}+00$ & 0 & $1.2 \mathrm{E}+07$ & 1 & $0.0 \mathrm{E}+00$ & 0 & $0.0 \mathrm{E}+00$ & 0 & $0.0 \mathrm{E}+00$ & 0 \\
\hline \multirow[t]{3}{*}{ MEG-4.1 (I213F) } & SIADFFLINKPK & $4.5 \mathrm{E}+05$ & 9 & $0.0 \mathrm{E}+00$ & 0 & $3.3 \mathrm{E}+07$ & 13 & $0.0 \mathrm{E}+00$ & 0 & $3.5 \mathrm{E}+08$ & 5 & $0.0 \mathrm{E}+00$ & 0 \\
\hline & $\begin{array}{l}\text { SIADFFLINKPKVPLWIVNPLY } \\
\text { YMVEK }\end{array}$ & $0.0 \mathrm{E}+00$ & 0 & $0.0 \mathrm{E}+00$ & 0 & $1.2 \mathrm{E}+07$ & 3 & $0.0 \mathrm{E}+00$ & 0 & $0.0 \mathrm{E}+00$ & 0 & $0.0 \mathrm{E}+00$ & 0 \\
\hline & median & $2.3 E+05$ & & $0.0 E+00$ & & $2.2 E+07$ & & $0.0 E+00$ & & $1.8 E+08$ & & $0.0 E+00$ & \\
\hline \multirow[t]{3}{*}{ MEG-4.2 (Full) } & FHEMPEYDDQLPDFPHK & $1.9 \mathrm{E}+06$ & $\underline{19}$ & $0.0 \mathrm{E}+00$ & 0 & $2.8 \mathrm{E}+08$ & $\underline{5}$ & $0.0 \mathrm{E}+00$ & 0 & $0.0 \mathrm{E}+00$ & 0 & $0.0 \mathrm{E}+00$ & 0 \\
\hline & QLEEEQNPFHK & $\overline{2.1 \mathrm{E}+05}$ & $\overline{10}$ & $0.0 \mathrm{E}+00$ & 0 & $\overline{1.3 \mathrm{E}+07}$ & $\overline{2}$ & $0.0 \mathrm{E}+00$ & 0 & $1.2 \mathrm{E}+07$ & 1 & $0.0 \mathrm{E}+00$ & 0 \\
\hline & median & $1.1 \mathrm{E}+06$ & & $0.0 \mathrm{E}+00$ & & $1.4 \mathrm{E}+08$ & & $0.0 \mathrm{E}+00$ & & $6.0 \mathrm{E}+06$ & & $0.0 \mathrm{E}+00$ & \\
\hline MEG-4.2 (Q67del) & QLEEENPFHK & $2.5 \mathrm{E}+05$ & 2 & $0.0 \mathrm{E}+00$ & 0 & $0.0 \mathrm{E}+00$ & 0 & $0.0 \mathrm{E}+00$ & 0 & $0.0 \mathrm{E}+00$ & 0 & $0.0 \mathrm{E}+00$ & 0 \\
\hline MEG-5 & SGGSSDISDMFGQNK & $4.9 \mathrm{E}+04$ & 3 & $4.9 \mathrm{E}+04$ & 4 & $7.2 \mathrm{E}+06$ & 1 & $6.6 \mathrm{E}+06$ & 1 & $0.0 \mathrm{E}+00$ & 0 & $0.0 \mathrm{E}+00$ & 0 \\
\hline
\end{tabular}




\begin{tabular}{|c|c|c|c|c|c|c|c|c|c|c|c|c|c|}
\hline \multirow[t]{5}{*}{ MEG-8.1 } & LPNPSQK & $3.7 \mathrm{E}+05$ & 2 & $0.0 \mathrm{E}+00$ & 0 & $0.0 \mathrm{E}+00$ & 0 & $0.0 \mathrm{E}+00$ & 0 & $0.0 \mathrm{E}+00$ & 0 & $0.0 \mathrm{E}+00$ & 0 \\
\hline & RVGDGFFDLFSEQEFHPINHK & $0.0 \mathrm{E}+00$ & 0 & $0.0 \mathrm{E}+00$ & 0 & $0.0 \mathrm{E}+00$ & 0 & $0.0 \mathrm{E}+00$ & 0 & $1.9 \mathrm{E}+07$ & 1 & $0.0 \mathrm{E}+00$ & 0 \\
\hline & VGDGFFDLFSEQEFHPINHK & $0.0 \mathrm{E}+00$ & 0 & $0.0 \mathrm{E}+00$ & 0 & $0.0 \mathrm{E}+00$ & 0 & $0.0 \mathrm{E}+00$ & 0 & $5.6 \mathrm{E}+07$ & 1 & $0.0 \mathrm{E}+00$ & 0 \\
\hline & TSFLNLK & $0.0 \mathrm{E}+00$ & 0 & $0.0 \mathrm{E}+00$ & 0 & $0.0 \mathrm{E}+00$ & 0 & $0.0 \mathrm{E}+00$ & 0 & $3.9 \mathrm{E}+07$ & 1 & $0.0 \mathrm{E}+00$ & 0 \\
\hline & median & $0.0 E+00$ & & $0.0 E+00$ & & $0.0 E+00$ & & $0.0 E+00$ & & $2.9 \mathrm{E}+07$ & & $0.0 \mathrm{E}+00$ & \\
\hline \multirow[t]{11}{*}{ MEG-8.2 } & MNTTPVHQEEPSFWR & $0.0 \mathrm{E}+00$ & 0 & $0.0 \mathrm{E}+00$ & 0 & $1.1 \mathrm{E}+07$ & 1 & $0.0 \mathrm{E}+00$ & 0 & $0.0 \mathrm{E}+00$ & 0 & $0.0 \mathrm{E}+00$ & 0 \\
\hline & $\begin{array}{l}\text { RMWNSFTSMFGSSDSSSGTN } \\
\text { NK }\end{array}$ & $3.0 \mathrm{E}+05$ & 9 & $0.0 \mathrm{E}+00$ & 0 & $2.8 \mathrm{E}+07$ & 3 & $0.0 \mathrm{E}+00$ & 0 & $0.0 \mathrm{E}+00$ & 0 & $0.0 \mathrm{E}+00$ & 0 \\
\hline & $\underline{\text { MWNSFTSMFGSSDSSSGTNNK }}$ & $\underline{6.0 \mathrm{E}+05}$ & $\underline{21}$ & $0.0 \mathrm{E}+00$ & 0 & $5.1 \mathrm{E}+07$ & 2 & $0.0 \mathrm{E}+00$ & 0 & $0.0 \mathrm{E}+00$ & 0 & $0.0 \mathrm{E}+00$ & 0 \\
\hline & $\frac{\text { MWNSFTSMFGSSDSSSGTNNK }}{\underline{\text { DTK }}}$ & $\underline{9.6 \mathrm{E}+05}$ & $\underline{14}$ & $0.0 \mathrm{E}+00$ & 0 & $1.3 \mathrm{E}+08$ & 3 & $0.0 \mathrm{E}+00$ & 0 & $0.0 \mathrm{E}+00$ & 0 & $0.0 \mathrm{E}+00$ & 0 \\
\hline & SPNPNTTEAK & $2.7 \mathrm{E}+04$ & 4 & $0.0 \mathrm{E}+00$ & 0 & $0.0 \mathrm{E}+00$ & 0 & $0.0 \mathrm{E}+00$ & 0 & $0.0 \mathrm{E}+00$ & 0 & $0.0 \mathrm{E}+00$ & 0 \\
\hline & SLSLKER & $4.4 \mathrm{E}+05$ & 8 & $1.7 \mathrm{E}+05$ & 3 & $0.0 \mathrm{E}+00$ & 0 & $0.0 \mathrm{E}+00$ & 0 & $9.1 \mathrm{E}+07$ & 1 & $7.9 \mathrm{E}+07$ & 1 \\
\hline & FNSIFGEEEYNPPK & $0.0 \mathrm{E}+00$ & 0 & $0.0 \mathrm{E}+00$ & 0 & $0.0 \mathrm{E}+00$ & 0 & $0.0 \mathrm{E}+00$ & 0 & $5.3 \mathrm{E}+07$ & 2 & $0.0 \mathrm{E}+00$ & 0 \\
\hline & FNSIFGEEEYNPPKDSDFTER & $0.0 \mathrm{E}+00$ & 0 & $0.0 \mathrm{E}+00$ & 0 & $0.0 \mathrm{E}+00$ & 0 & $0.0 \mathrm{E}+00$ & 0 & $6.1 \mathrm{E}+05$ & 1 & $0.0 \mathrm{E}+00$ & 0 \\
\hline & LWMLFK & $0.0 \mathrm{E}+00$ & 0 & $0.0 \mathrm{E}+00$ & 0 & $0.0 \mathrm{E}+00$ & 0 & $0.0 \mathrm{E}+00$ & 0 & $2.8 \mathrm{E}+07$ & $\underline{2}$ & $0.0 \mathrm{E}+00$ & 0 \\
\hline & $\overline{\text { HCFLNFK }}$ & $0.0 \mathrm{E}+00$ & 0 & $0.0 \mathrm{E}+00$ & 0 & $0.0 \mathrm{E}+00$ & 0 & $0.0 \mathrm{E}+00$ & 0 & $\overline{5.3 \mathrm{E}+07}$ & $\overline{1}$ & $0.0 \mathrm{E}+00$ & 0 \\
\hline & median & $1.4 \mathrm{E}+04$ & & $\mathbf{0 . 0 E}+00$ & & $\mathbf{0 . 0 E}+00$ & & $\mathbf{0 . 0 E}+00$ & & $3.1 \mathrm{E}+05$ & & $\mathbf{0 . 0 E}+00$ & \\
\hline MEG-8.4 & IIQNDHALK & $0.0 \mathrm{E}+00$ & 0 & $0.0 \mathrm{E}+00$ & 0 & $8.9 \mathrm{E}+06$ & 2 & $0.0 \mathrm{E}+00$ & 0 & $0.0 \mathrm{E}+00$ & 0 & $0.0 \mathrm{E}+00$ & 0 \\
\hline MEG-12 & AYGIWSLFSYFYK & $0.0 \mathrm{E}+00$ & 0 & $0.0 \mathrm{E}+00$ & 0 & $0.0 \mathrm{E}+00$ & 0 & $0.0 \mathrm{E}+00$ & 0 & $3.9 \mathrm{E}+06$ & 1 & $0.0 \mathrm{E}+00$ & 0 \\
\hline $\begin{array}{l}\text { MEG-14, } \\
\text { proteoforms } 1 \\
(\text { AOL57989.1), } 3 \\
(\text { AOL57991.1) and } 9 \\
(\text { OOL57997.1) }\end{array}$ & $\begin{array}{l}\text { THGATSTSTHGATSTAKPAAS } \\
\text { TPPK }\end{array}$ & $2.7 \mathrm{E}+05$ & 2 & $0.0 \mathrm{E}+00$ & 0 & $1.9 \mathrm{E}+07$ & 10 & $0.0 \mathrm{E}+00$ & 0 & $1.2 \mathrm{E}+06$ & 1 & $0.0 \mathrm{E}+00$ & 0 \\
\hline $\begin{array}{l}\text { MEG-14, } \\
\text { proteoforms } 5 \\
(\text { AOL57993.1) and } 6 \\
(\text { AOL57994.1) }\end{array}$ & $\begin{array}{l}\text { THGATSTTHGATSTAKPAAST } \\
\text { PPK }\end{array}$ & $0.0 \mathrm{E}+00$ & 0 & $0.0 \mathrm{E}+00$ & 0 & $3.7 \mathrm{E}+06$ & 1 & $0.0 \mathrm{E}+00$ & 0 & $0.0 \mathrm{E}+00$ & 0 & $0.0 \mathrm{E}+00$ & 0 \\
\hline $\begin{array}{l}\text { MEG-14, } \\
\text { proteoforms } 1 \\
(\text { AOL57989.1), } 2 \\
(\text { AOL57990.1), } 3\end{array}$ & AAATSTIKPTVTTPK & $5.7 \mathrm{E}+05$ & 13 & $0.0 \mathrm{E}+00$ & 0 & $1.0 \mathrm{E}+08$ & 9 & $0.0 \mathrm{E}+00$ & 0 & $0.0 \mathrm{E}+00$ & 0 & $0.0 \mathrm{E}+00$ & 0 \\
\hline
\end{tabular}


(AOL57991.1), 4

(AOL57992.1), 5

(AOL57993.1), 6

(AOL57994.1) and 9

(AOL57997.1)

MEG-14,

proteoforms 4

(AOL57992.1), 6

(AOL57994.1) and

7(AOL57995.1)

MEG-14,

proteoforms 2

(AOL57990.1) and

10 (AOL57998.1)

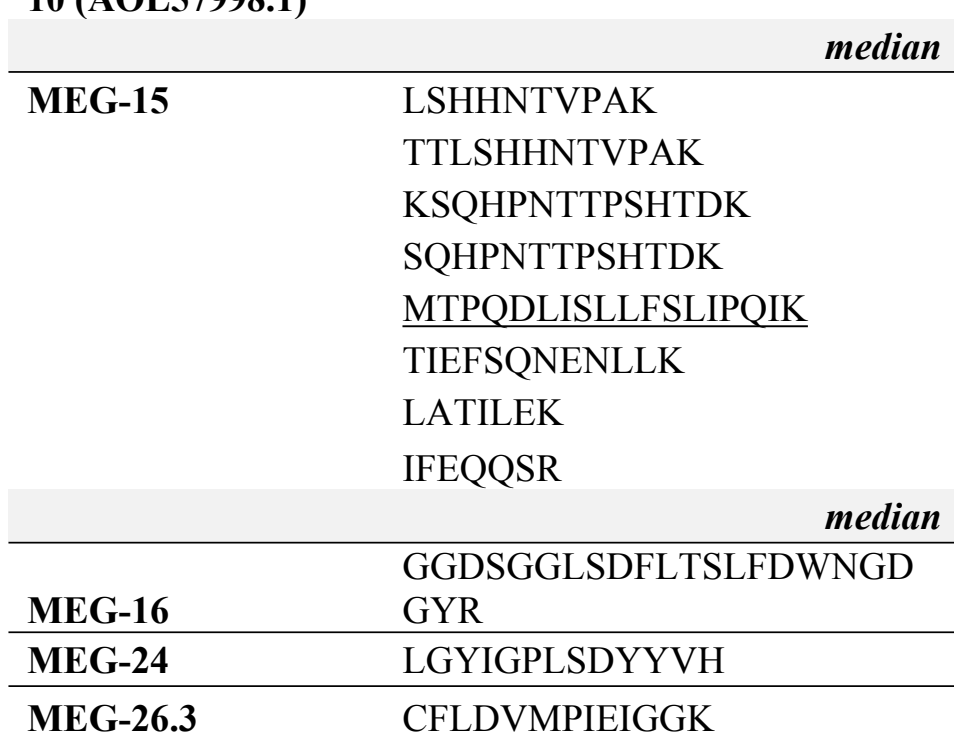

AAATSTTEPTVTTSKPSPAKPA

ASNTAKPAASTPK

AAATSTTEPTVTTKPSPAKPAA

SNTAKPAASTPK

CFLDVMPIEIGGK

\begin{tabular}{|c|c|c|c|c|c|c|c|}
\hline $2.7 \mathrm{E}+07$ & 2 & $0.0 \mathrm{E}+00$ & 0 & $0.0 \mathrm{E}+00$ & 0 & $0.0 \mathrm{E}+00$ & 0 \\
\hline $4.0 \mathrm{E}+07$ & 1 & $0.0 \mathrm{E}+00$ & 0 & $0.0 \mathrm{E}+00$ & 0 & $0.0 \mathrm{E}+00$ & 0 \\
\hline $2.7 E+07$ & & $0.0 \mathrm{E}+00$ & & $0.0 \mathrm{E}+00$ & & $0.0 \mathrm{E}+00$ & \\
\hline $3.8 \mathrm{E}+07$ & 8 & $0.0 \mathrm{E}+00$ & 0 & $2.2 \mathrm{E}+06$ & 1 & $0.0 \mathrm{E}+00$ & 0 \\
\hline $1.1 E+09$ & 6 & $0.0 \mathrm{E}+00$ & 0 & $5.6 \mathrm{E}+06$ & 2 & $0.0 \mathrm{E}+00$ & 0 \\
\hline $0.0 \mathrm{E}+00$ & 0 & $0.0 \mathrm{E}+00$ & 0 & $0.0 \mathrm{E}+00$ & 0 & $0.0 \mathrm{E}+00$ & 0 \\
\hline $0.0 \mathrm{E}+00$ & 0 & $0.0 \mathrm{E}+00$ & 0 & $0.0 \mathrm{E}+00$ & 0 & $0.0 \mathrm{E}+00$ & 0 \\
\hline $0.0 \mathrm{E}+00$ & 0 & $0.0 \mathrm{E}+00$ & 0 & $\underline{1.8 \mathrm{E}+07}$ & $\underline{3}$ & $0.0 \mathrm{E}+00$ & 0 \\
\hline $0.0 \mathrm{E}+00$ & 0 & $0.0 \mathrm{E}+00$ & 0 & $3.4 \mathrm{E}+08$ & 1 & $0.0 \mathrm{E}+00$ & 0 \\
\hline $0.0 \mathrm{E}+00$ & 0 & $0.0 \mathrm{E}+00$ & 0 & $1.5 \mathrm{E}+08$ & 1 & $0.0 \mathrm{E}+00$ & 0 \\
\hline $0.0 \mathrm{E}+00$ & 0 & $0.0 \mathrm{E}+00$ & 0 & $2.1 \mathrm{E}+07$ & 1 & $0.0 \mathrm{E}+00$ & 0 \\
\hline $0.0 E+00$ & & $0.0 \mathrm{E}+00$ & & $1.2 \mathrm{E}+07$ & & $0.0 \mathrm{E}+00$ & \\
\hline $0.0 \mathrm{E}+00$ & 0 & $0.0 \mathrm{E}+00$ & 0 & $7.7 \mathrm{E}+04$ & 1 & $0.0 \mathrm{E}+00$ & 0 \\
\hline $3.0 \mathrm{E}+07$ & 4 & $3.6 \mathrm{E}+07$ & 2 & $1.3 \mathrm{E}+08$ & 1 & $1.3 \mathrm{E}+08$ & 1 \\
\hline $8.8 \mathrm{E}+06$ & 1 & $0.0 \mathrm{E}+00$ & 0 & $0.0 \mathrm{E}+00$ & 0 & $0.0 \mathrm{E}+00$ & 0 \\
\hline
\end{tabular}

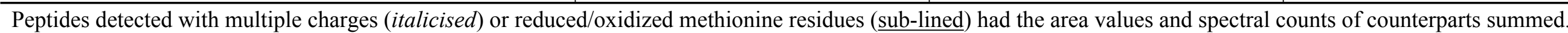




\section{Supplementary Material S4_Descriptive statistics of QconCAT assay and protein copy per cell estimation}

Peptide statistics, optimal isotopic dilution and peak verification by mProphet

\begin{tabular}{|c|c|c|c|c|c|c|c|c|c|}
\hline $\begin{array}{l}\text { GeneDB } \\
\text { acession }\end{array}$ & Description & Peptide & $\begin{array}{c}\text { Best } \\
\text { dilution } \\
\text { point of } \\
\text { EsoCAT }\end{array}$ & $\begin{array}{c}\text { Peak } \\
\text { detection } \\
\text { by } \\
\text { mProphet }\end{array}$ & $\begin{array}{l}\text { Peptide } \\
\text { type }\end{array}$ & $\begin{array}{l}\text { \# valid } \\
\text { replicates }\end{array}$ & $\begin{array}{l}\text { Average } \\
\text { (fmol) }\end{array}$ & SD & CV \\
\hline \multirow[t]{3}{*}{ Smp_132480 } & \multirow[t]{3}{*}{ Aspartyl protease } & NIFSLDYFTSK & $100 x$ & $\overline{T R U E}$ & A1 & 4 & 0.73 & 0.07 & $9.61 \%$ \\
\hline & & INSLVIPNQAFGEMR & $100 x$ & TRUE & A1 & & & & \\
\hline & & INSLVIPNQAFGEM[+16]R & $100 x$ & TRUE & A1 & 4 & 2.17 & 0.19 & $8.55 \%$ \\
\hline \multirow[t]{2}{*}{ Smp_151220 } & \multirow{2}{*}{$\begin{array}{l}\beta-1,3-\mathrm{N}- \\
\text { galactosyltransferase }\end{array}$} & NAENPFDSPYYR & $100 x$ & TRUE & A1 & 4 & 1.22 & 0.09 & $7.63 \%$ \\
\hline & & QVPLYLFIDDDYDLVPK & - & FALSE & B & - & - & - & - \\
\hline \multirow[t]{2}{*}{ Smp_142970 } & \multirow[t]{2}{*}{ Palmitoyl thioesterase } & LATAIQNEIPR & $100 x$ & TRUE & A1 & 4 & 2.77 & 0.20 & $7.19 \%$ \\
\hline & & VGTVVSIGGPQQGIFGIPK & $10 \mathrm{X}$ & TRUE & A1 & 4 & 2.00 & 0.19 & $9.52 \%$ \\
\hline \multirow[t]{2}{*}{ Smp_242990 } & \multirow[t]{2}{*}{ VAL-7 } & VFVVC[+57]NYGPGGK & $10 x$ & TRUE & A1 & 4 & 61.66 & 5.70 & $9.25 \%$ \\
\hline & & WTYVGQSIAFVPK & $10 x$ & TRUE & A1 & 4 & 16.86 & 1.77 & $10.49 \%$ \\
\hline \multirow[t]{2}{*}{ Smp_163630 } & \multirow[t]{2}{*}{ MEG-4.1 } & QINDGTSDKPK & $1 x$ & TRUE & A1 & 3 & 20.69 & 0.45 & $2.18 \%$ \\
\hline & & SIADIFLINKPK & $10 x$ & TRUE & A1 & 4 & 14.70 & 2.43 & $16.50 \%$ \\
\hline Smp_085840 & MEG-4.2 & QLEEEQNPFHK & $10 x$ & TRUE & A1 & 4 & 19.00 & 2.48 & $13.07 \%$ \\
\hline \multirow[t]{3}{*}{ Smp_172180 } & \multirow[t]{3}{*}{ MEG-8.2 } & FNSIFGEEEYNPPK & $100 x$ & TRUE & A1 & 3 & 3.06 & 0.12 & $3.96 \%$ \\
\hline & & MNTTPVHQEEPSFWR & $100 x$ & TRUE & A1 & 2 & 122 & 017 & \\
\hline & & M[+16]NTTPVHQEEPSFWR & $100 x$ & TRUE & A1 & 3 & 1.23 & 0.17 & $13.89 \%$ \\
\hline \multirow[t]{3}{*}{ Smp_152630 } & \multirow[t]{3}{*}{ MEG-12 } & ENYEQQLQQPK & $1 x$ & TRUE & A1 & 4 & 593.10 & 39.99 & $6.74 \%$ \\
\hline & & AYGIWSLFSYFYK & - & FALSE & $\mathrm{B}$ & - & - & - & - \\
\hline & & FHIVFFCGENYEQQLQQPK & - & FALSE & $\mathrm{B}$ & - & - & - & - \\
\hline \multirow[t]{3}{*}{ Smp_010550 } & \multirow[t]{3}{*}{ MEG-15 } & TIEFSQNENLLK & $100 x$ & TRUE & A1 & 4 & 2.41 & 0.22 & $9.08 \%$ \\
\hline & & TTLSHHNTVPAK & $100 x$ & TRUE & A1 & 4 & 3.84 & 0.38 & $9.80 \%$ \\
\hline & & LSHHNTVPAK 100x & $10 x$ & FALSE & $\mathrm{A} 2$ & 4 & - & - & - \\
\hline
\end{tabular}




\section{Equation to determine protein copies per cell (cpc)}

$$
c p c=\frac{\text { Measured protein concentration }[\mathrm{fmol} / \mu \mathrm{g} \text { protein }] \times \text { Avogadro's constant }}{\text { Number of gland cells per fragment } \div \text { Protein content per ESO fragment }(\mu \mathrm{g})}
$$

OBS: The number of cells in the Schistosoma mansoni esophageal glands was previously described by Li XH et al. (2013) ${ }^{9}$. Then, we estimated the number of cells per ug of ESO based on the total protein content of individual fragments $0.51 \pm 0.17 \mu \mathrm{g} / \mathrm{ESO} *$, as follows:

\begin{tabular}{|c|c|c|}
\hline & $\begin{array}{c}\text { Gland cells per } \\
\text { esophageal fragment }\end{array}$ & $\begin{array}{c}\text { Gland cells per } \mu \mathrm{g} \text { of } \\
\text { ESO* }\end{array}$ \\
\hline Anterior oesophageal gland (Anterior ESO) & 750 & 1456.3 \\
\hline Posterior oesophageal gland (Posterior ESO) & 1000 & 1941.7 \\
\hline
\end{tabular}

QconCAT quantification expressed in protein copies per cell

\begin{tabular}{|c|c|c|c|}
\hline $\begin{array}{l}\text { GeneDB } \\
\text { accession }\end{array}$ & Description (site of expression) & 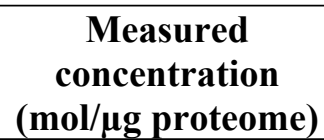 & $\begin{array}{l}\text { Protein copies } \\
\text { per esophageal } \\
\text { gland cell }\end{array}$ \\
\hline Smp_163630 & MEG-4.1 (Posterior ESO) - canonical form & $2.07 \mathrm{E}-14$ & $6,416,803$ \\
\hline Smp_163630 & proteoform subset & $1.47 \mathrm{E}-14$ & $4,559,062$ \\
\hline Smp_085840 & MEG-4.2 (Posterior ESO) & $1.90 \mathrm{E}-14$ & $5,892,665$ \\
\hline Smp_172180 & MEG-8.2 (Posterior ESO) & $2.15 \mathrm{E}-15$ & 665,251 \\
\hline Smp_152630 & MEG-12 (Anterior ESO) & $5.93 \mathrm{E}-13$ & $245,258,939$ \\
\hline Smp_010550 & MEG-15(Posterior ESO) & $3.12 \mathrm{E}-15$ & 968,568 \\
\hline Smp_242990 & VAL-7 (Posterior ESO) & 3.93E-14 & $12,176,108$ \\
\hline Smp_142970 & Palmitoyl thioesterase (Posterior ESO) & $2.38 \mathrm{E}-15$ & 738,444 \\
\hline Smp_132480 & Aspartyl protease (Posterior ESO) & $1.45 \mathrm{E}-15$ & 449,393 \\
\hline Smp_151220 & $\beta-1,3-\mathrm{N}$-galactosyltransferase (Posterior ESO) & $1.22 \mathrm{E}-15$ & 377,751 \\
\hline
\end{tabular}

${ }^{9}$ Li X-H, de Castro-Borges W, Parker-Manuel S, Vance GM, DeMarco R, Neves LX, et al. (2013) The Schistosome Oesophageal Gland: Initiator of Blood Processing. PLoS Negl Trop Dis 7(7): e2337.

https://doi.org/10.1371/journal.pntd.0002337 


\section{Supplementary Material S5_Abundance of gastrodermis markers and statistical differences}

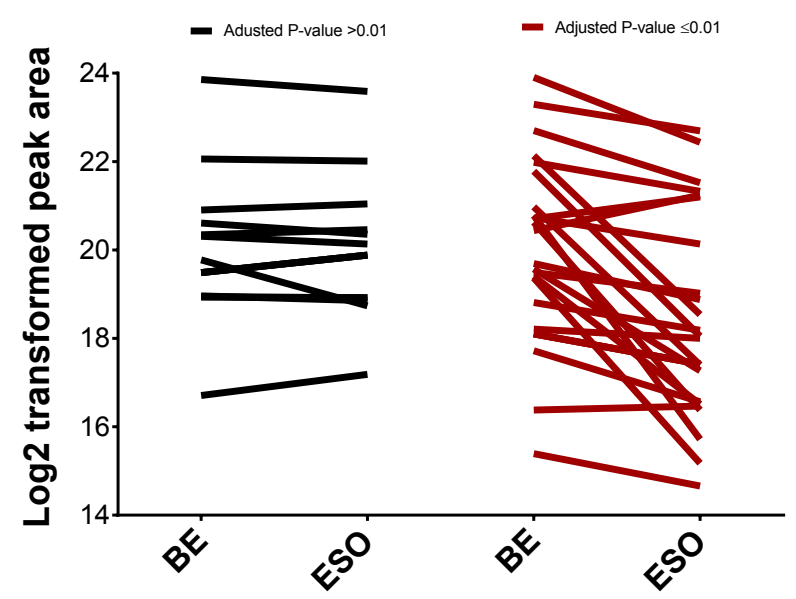

Proteins differentially abundant (Benjamin-Hochberg adjusted $p$-values $\leq 0.01$; red lines) and those more evenly distributed in the samples spanned over the same dynamic range. Either presence or absence of differential expression between ESO and BE was not biased by the MS-signal intensity (i.e. favouring high abundant proteins).

OBS: Saposin B (Smp_105420) was detected enriched in the BE sample supported by 1 unique peptide. A representative annotated spectrum and quantitative data is displayed below.

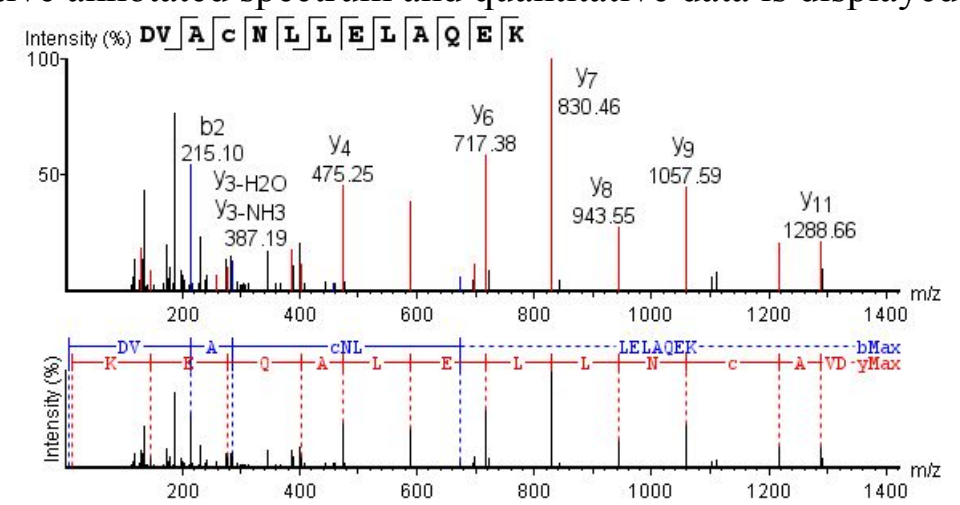

Protein List

Protein Accession Contains:

Protein Description Contains: liberian

Protein Ptm Contains:

\begin{tabular}{|l|l|l|l|l|l|l|l|l|l|l|l|}
\hline Protein Group & Protein ID & Accession & Significance & Coverage (\%) & \#Peptides & \#Unique & PTM & Avg. Mass & Sample Profile & Group Profile & Description
\end{tabular}

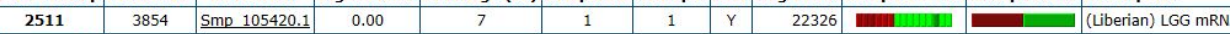

total 1 proteins

Smp_105420.1

back to list

| Protein Coverage | Supporting Peptides |

Protein Coverage:

MFGFFILTIF CITGFVTSIN QPELEFGYKD VACNLLELAQ EKSLQIMKEE QFIGNMVEYL VSITCDNVKD LNKRLQCKTS

c Carbamidomethylation (+57.02)

81 MATEARILVQ YFIEFIESYR LKTMLNWCQS TLERPQYGNS SFLCSTCEMA VAYLKTFSKS EEAKAIVHQA VDKICSLTGS

161 FEVQCSFLGG MFIDKYIDTI STMDPDSACM TMHMCS

Supporting Peptides:

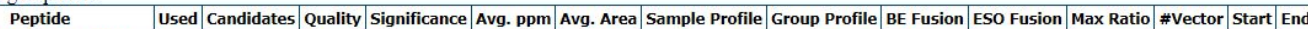

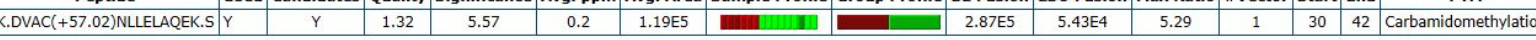
total 1 peptides 